\title{
The second lightest CP-even Higgs boson signals in the NMSSM at the LHC
}

\author{
M. M. Almarashi \\ Physics Department, Faculty of Science, Taibah University, \\ P.O. Box 344, Al-Madinah Al-Munawwarah, Saudi Arabia \\ al_marashi@hotmail.com
}

Received 10 February 2018

Revised 28 March 2018

Accepted 1 April 2018

Published 5 April 2018

\begin{abstract}
We study the signal rates of the second lightest CP-even Higgs boson, $h_{2}$, of the NMSSM produced in gluon fusion, in association with bottom quarks and in association with top quarks, which is not the SM-like Higgs boson, at the LHC. We evaluate the production rates of $h_{2}$ in the SM fermionic and bosonic final states in addition to $a_{1} a_{1}, h_{1} h_{1}$ and $Z a_{1}$ final states. It is observed that the size of the signal rates in some regions of the NMSSM parameter space is quite large and that could help extracting $h_{2}$ signals at the LHC through a variety of decay channels.
\end{abstract}

Keywords: NMSSM; CP-even Higgs boson; Higgs signals.

PACS numbers: 14.80.Da, 12.60.Fr, 14.80.Ec

\section{Introduction}

Assuming CP-conservation in the Higgs sector, the Next-to-Minimal Supersymmetric Standard Model (NMSSM) $)^{1-11}$ predicts one or more light CP-even Higgs boson, either the lightest Higgs boson $h_{1}$ or the second lightest Higgs boson $h_{2}$, which could be the Higgs particle with a mass around $125 \mathrm{GeV}$ discovered by the Large Hadron Collider (LHC) $.12-15$ This discovered SM-like Higgs can be accommodated in the context of the NMSSM without much fine tuning, see Refs. $16-33$ for more details.

This is an Open Access article published by World Scientific Publishing Company. It is distributed under the terms of the Creative Commons Attribution 4.0 (CC-BY) License. Further distribution of this work is permitted, provided the original work is properly cited. 
Within the NMSSM, the Higgs field consists of the two MSSM-type Higgs doublets in addition to the Higgs singlet, giving rise to seven Higgs states: three CP-even Higgses $h_{1,2,3}\left(m_{h_{1}}<m_{h_{2}}<m_{h_{3}}\right)$, two CP-odd Higgses $a_{1,2}\left(m_{a_{1}}<m_{a_{2}}\right)$ and a pair of charged Higgses $h^{ \pm}$. When the singlet field acquires a vacuum expectation value $(\mathrm{VEV})$, an "effective" $\mu$-term, $\mu_{\text {eff }}$, is generated automatically, which is naturally of the order of the electroweak scale. $\stackrel{4}{ }$ Moreover, due to the presence of the singlet field, the phenomenology of the NMSSM Higgs sector is richer than that of the MSSM, which has only five Higgs states: $h, H, A$ and $H^{ \pm}$. The phenomenology of the NMSSM Higgs sector has been studied by many authors, for a review and details, see Refs. $\underline{34}-36$ and references therein. For the latest studies about the NMSSM Higgs sector, see Refs. 37-40.

Following the discovery of the SM-like Higgs boson at the LHC, looking for other Higgs bosons at the LHC, if they exist, would point to the existence of supersymmetric models. Searches for additional heavy neutral Higgs bosons have been carried out by the ATLAS and CMS Collaborations in $b \bar{b},{ }^{41,42} \tau \tau,{ }^{43-50}$ and gauge boson, $W W$ and $Z Z, \underline{51,52}$ final states. No signal has been found so far, putting some constraints on such Higgs bosons.

In this paper, we study the production rates of the second lightest Higgs boson $h_{2}$, if it is not the SM-like Higgs boson, of the NMSSM at the LHC through the following production channels: gluon fusion $g g \rightarrow h_{2}$ and associated production with bottom-antibottom pair $g g \rightarrow b \bar{b} h_{2}$ and top-antitop pair $g g \rightarrow t \bar{t} h_{2}$ for the most promising final states. We calculate the inclusive cross-section of $h_{2}$. It is observed that there exist some regions of the NMSSM parameter space where the cross-section times branching ratios are quite sizable and could help extracting $h_{2}$ signals at the LHC through the above production channels.

The paper is organized as follows. In Sec. 2, we briefly discuss the NMSSM Higgs sector, describing the NMSSM parameter space scans performed and the allowed decay channels of $h_{2}$. In Sec. 3, we present the inclusive event rates of $h_{2}$ production at the LHC for various decay channels. Finally, we summarize our results in Sec. 4 .

\section{The Higgs Sector of the NMSSM}

The scale-invariant superpotential of the NMSSM, including only the third generation fermions, is given by

$$
W=h_{t} \hat{Q} \hat{H}_{u} \hat{t}_{R}^{c}-h_{b} \hat{Q} \hat{H}_{d} \hat{b}_{R}^{c}-h_{\tau} \hat{L} \hat{H}_{d} \hat{\tau}_{R}^{c}+\lambda \hat{S} \hat{H}_{u} \hat{H}_{d}+\frac{1}{3} \kappa \hat{S}^{3}
$$

where $h_{t}, h_{b}, h_{\tau}, \lambda$ and $\kappa$ are dimensionless couplings. The term $\lambda \hat{S} \hat{H}_{u} \hat{H}_{d}$ is required to solve the $\mu$-problem. .53 The last term, which is cubic in the singlet superfield, has been introduced to break the Peccei-Quinn (PQ) symmetry. ${ }^{44,55}$ 
The soft SUSY-breaking potential containing only the Higgs fields is given by

$$
\begin{aligned}
V_{\text {soft }}= & m_{H_{u}}^{2}\left|H_{u}\right|^{2}+m_{H_{d}}^{2}\left|H_{d}\right|^{2}+m_{S}^{2}|S|^{2} \\
& +\left(\lambda A_{\lambda} S H_{u} H_{d}+\frac{1}{3} \kappa A_{\kappa} S^{3}+\text { h.c. }\right),
\end{aligned}
$$

where $A_{\lambda}$ and $A_{\kappa}$ are dimensionful parameters of the order of the mass scale of supersymmetric particles.

The NMSSM Higgs sector is fully described by six independent parameters: $\kappa$, $A_{\kappa}, \lambda, A_{\lambda}, \tan \beta$ (the ratio of the VEVs of the two Higgs doublets, $H_{u}$ and $H_{d}$ ) and $\mu_{\mathrm{eff}}=\lambda\langle S\rangle$ (where $\langle S\rangle$ is the VEV of the singlet field).

For our study of the NMSSM Higgs sector, we have used the package NMSSMTools5.1.2 $2^{56-58}$ to compute the masses, couplings and decay widths of all the NMSSM Higgs bosons. The package systematically takes into account various theoretical and experimental constraints. We use the package to scan over some regions of the NMSSM parameter space in order to obtain a general view of the phenomenology of the second lightest CP-even Higgs boson, $h_{2}$, at the LHC. We have set the six tree level parameters in the following ranges:

$$
\begin{gathered}
0.001 \leq \lambda \leq 0.1, \quad-0.1 \leq \kappa \leq 0.1, \quad 1.6 \leq \tan \beta \leq 60 \\
100 \leq \mu \leq 1000 \mathrm{GeV}, \quad-1000 \leq A_{\lambda} \leq 1000 \mathrm{GeV}, \quad-1000 \leq A_{\kappa} \leq 1000 \mathrm{GeV} .
\end{gathered}
$$

Note that we focus here on scenario with small values of $\lambda$ and $\kappa$ in order to increase the probability of getting a lot of surviving data points with $m_{h_{2}} \lesssim 500 \mathrm{GeV}$.

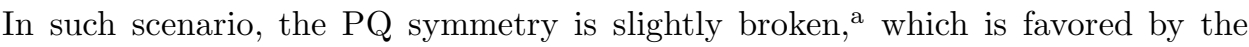
renormalization group flow from the grand unification scale down to the electroweak scale, see Ref. 59 for more details. Further information about the effect of $\lambda$ and $\kappa$ on the two lightest CP-even Higgs boson masses can be obtained from the following sum rule: ${ }^{59}$

$$
m_{h_{1}}^{2}+m_{h_{2}}^{2} \approx M_{Z}^{2}+\frac{1}{2} \kappa\langle S\rangle\left(4 \kappa\langle S\rangle+\sqrt{2} A_{\kappa}\right),
$$

where $\langle S\rangle=\sqrt{2} \mu / \lambda$.

Remaining terms, contributing at higher order level, which are soft SUSY breaking right- and left-handed masses for the first two generations and the third generation, soft SUSY breaking trilinear couplings and gaugino soft SUSY breaking masses have been set as

- $m_{Q}=m_{U}=m_{D}=m_{L}=m_{E}=m_{Q_{3}}=m_{U_{3}}=m_{D_{3}}=m_{L_{3}}=m_{E_{3}}=$ $1000 \mathrm{GeV}$,

- $A_{U_{3}}=A_{D_{3}}=A_{E_{3}}=2000 \mathrm{GeV}$,

- $M_{1}=250 \mathrm{GeV}, M_{2}=500 \mathrm{GeV}, M_{3}=1500 \mathrm{GeV}$.

a The PQ symmetry is slightly broken when $\kappa$ is small, i.e. $\kappa \ll 1$. 


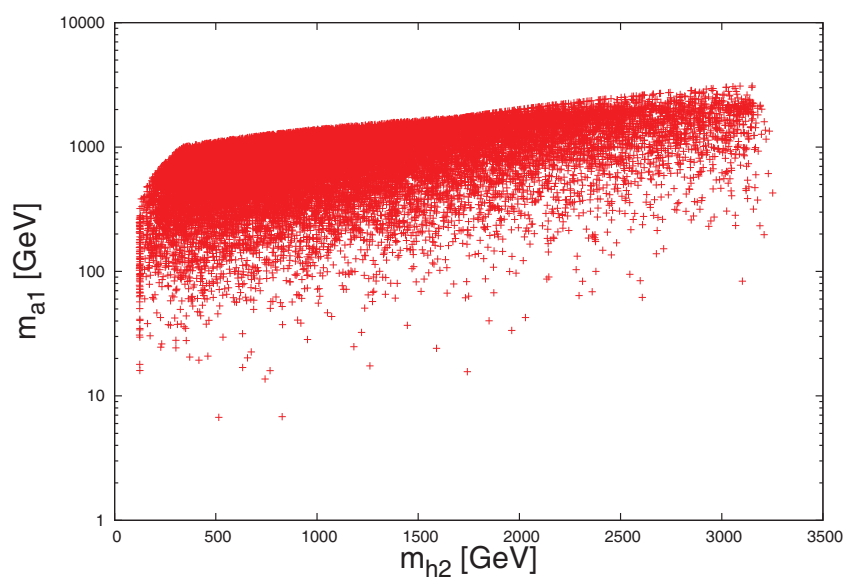

Fig. 1. The correlations between the second lightest CP-even Higgs mass, $m_{h_{2}}$ and the lightest CP-odd Higgs masses, $m_{a_{1}}$.

In our study for looking for $h_{2}$ at the LHC, we focus on the following decay channels:

$$
\begin{gathered}
\quad h_{2} \rightarrow \tau^{+} \tau^{-}, \quad h_{2} \rightarrow b \bar{b}, \quad h_{2} \rightarrow t \bar{t}, \quad h_{2} \rightarrow \gamma \gamma, \quad h_{2} \rightarrow Z \gamma, \\
h_{2} \rightarrow W^{+} W^{-}, \quad h_{2} \rightarrow Z Z, \quad h_{2} \rightarrow a_{1} a_{1}, \quad h_{2} \rightarrow h_{1} h_{1}, \quad h_{2} \rightarrow Z a_{1} .
\end{gathered}
$$

We perform a random scan over some fraction of the NMSSM parameter space. The output of the scan contains masses, branching ratios and couplings of both the NMSSM Higgses and SUSY particles for all the surviving data points which have passed the various experimental and theoretical constraints implemented in the package NMSSMTools5.1.2. $\underline{56} \underline{-58}$

\section{Higgs Boson Signal Rates}

In order to investigate the discovery prospects for $h_{2}$ at the $\mathrm{LHC},{ }^{\mathrm{b}}$ we calculate the cross-sections for $h_{2}$ production for the surviving data points by using CalcHEP. 60 We focus here on the following production channels:

(i) gluon fusion $g g \rightarrow h_{2}$;

(ii) associated production with a pair of bottom quarks $g g \rightarrow b \bar{b} h_{2}$;

(iii) associated production with a pair of top quarks $g g \rightarrow t \bar{t} h_{2} \cdot \stackrel{\mathrm{c}}{\mathrm{c}}$

Figure 1 displays the correlation between the lightest CP-odd Higgs boson mass $m_{a_{1}}$ and the second lightest CP-even Higgs boson mass $m_{h_{2}}$. It is shown that $a_{1}$ can be very light, $\sim 7 \mathrm{GeV} . \mathrm{d}$ It is also interesting to note that the smaller the $m_{h_{2}}$, the smaller the $m_{a_{1}}$. Furthermore, it is obvious that there are some points of the

\footnotetext{
${ }^{\mathrm{b}}$ We assume a center-of-mass energy $\sqrt{s}=14 \mathrm{TeV}$ for the LHC.

${ }^{c}$ The production modes $q \bar{q} \rightarrow b \bar{b} h_{2}$ and $q \bar{q} \rightarrow t \bar{t} h_{2}$ are negligible at the LHC with $\sqrt{s}=14 \mathrm{TeV}$.

${ }^{\mathrm{d}}$ For the mass region $m_{a_{1}}<2 m_{b}$, the decay into $\tau^{+} \tau^{-}$is dominant compared to the decay into $c \bar{c}$ or $\mu^{+} \mu^{-}$.
} 
NMSSM parameter space where the two Higgs states $h_{2}$ and $a_{1}$ can simultaneously have the same mass around $125 \mathrm{GeV}$.

As an initial step of our analysis, we show in Fig. $\underline{2}$ the cross-sections for $h_{2}$ produced in gluon fusion (left), in association with bottom quarks (middle) and in association with top quarks (right) against $\lambda$ (top) and $\kappa$ (bottom). It is clear that in our chosen parameter space, the positive values of $\kappa$ are favored, while the distributions in $\lambda$ are nearly uniform. Also, it is clear that the maximum values of cross-sections do not only occur in the region with nearly vanishing $\lambda$ and $\kappa$, MSSM-

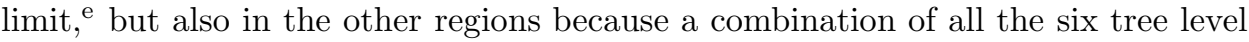
parameters jointly affects $m_{h_{2}}$, which in turn affects the values of cross-sections. The maximum cross-sections occur when the $h_{2}$ becomes more doublet-like than the singlet-like.

As a second step, we calculate the total cross-section times branching ratios of $h_{2}$ production at the LHC in femtobarn $(\mathrm{fb})$ :

$$
\begin{aligned}
& \sigma\left(g g \rightarrow h_{2}\right) \times \operatorname{Br}\left(h_{2} \rightarrow X X\right), \\
& \sigma\left(g g \rightarrow b \bar{b} h_{2}\right) \times \operatorname{Br}\left(h_{2} \rightarrow X X\right), \\
& \sigma\left(g g \rightarrow t \bar{t} h_{2}\right) \times \operatorname{Br}\left(h_{2} \rightarrow X X\right),
\end{aligned}
$$

where $h_{2} \rightarrow X X$ represents $h_{2}$ decay channels with $X X=\tau^{+} \tau^{-}, b \bar{b}, t \bar{t}, \gamma \gamma, Z \gamma$, $W^{+} W^{-}, Z Z, a_{1} a_{1}, h_{1} h_{1}$ and $Z a_{1}$.

Figure $\underline{3}$ displays the signal rates for $h_{2}$ produced in gluon fusion (left), in association with bottom quarks (middle) and in association with top quarks (right) for $\tau^{+} \tau^{-}$(top), $b \bar{b}$ (middle) and $t \bar{t}$ (bottom) final states as functions of $m_{h_{2}}$. We note that there is a gap in the figure with only few points between the upper region and lower region of the parameter space in the mass range $m_{h_{2}} \gtrsim 480 \mathrm{GeV}$. This is because the decay $h_{2} \rightarrow a_{1} a_{1}$, which is dominant in large area of the NMSSM parameter space, is closed or suppressed in the upper region. It is clear from the figure that the cross-section times branching ratio for the gluon fusion process is the highest one in general, reaching up to $2600 \mathrm{fb}, 26,000 \mathrm{fb}$ and $60 \mathrm{fb}$ for $\tau^{+} \tau^{-}$, $b \bar{b}$ and $t \bar{t}$ final states, respectively, see the left panels of the figure. The inclusive cross-section for the production in association with bottom quarks is the second largest one, topping $30 \mathrm{fb}, 300 \mathrm{fb}$ and $25 \mathrm{fb}$ in case of $\tau^{+} \tau^{-}, b \bar{b}$ and $t \bar{t}$ final states, respectively, see the middle panels of the figure. ${ }_{-}^{\mathrm{f}}$ However, there is some region

\footnotetext{
e The MSSM limit can be obtained by simultaneously taking $\lambda \rightarrow 0$ and $\kappa \rightarrow 0$ while keeping the parameters $\mu_{\mathrm{eff}}, A_{\lambda}$ and $A_{\kappa}$ fixed, which is not applied in our study.

${ }_{\mathrm{f}}$ Searches at the LHC for additional neutral MSSM Higgs bosons set some limits for the production of such bosons. For $\tau$ lepton final states, these range from $18,000 \mathrm{fb}$ at $90 \mathrm{GeV}$ to $3.5 \mathrm{fb}$ at $3.2 \mathrm{TeV}$ for production via gluon fusion and from $15,000 \mathrm{fb}$ at $90 \mathrm{GeV}$ to $2.5 \mathrm{fb}$ at $3.2 \mathrm{TeV}$ for production in association with $b$ quarks at a center-of-mass energy of $13 \mathrm{TeV}$, corresponding to an integrated luminosity of $35.9 \mathrm{fb}^{-1}$, see Ref. 50 . For $b$ quark final states, the upper limits on Higgs boson produced in association with at least one additional $b$ quark range from about $250,000 \mathrm{fb}$ at $100 \mathrm{GeV}$ to about $1000 \mathrm{fb}$ at $900 \mathrm{GeV}$ at a center-of-mass energy of $8 \mathrm{TeV}$ at the LHC, corresponding to an integrated luminosity of $19.7 \mathrm{fb}^{-1}$, see Ref. 42 .
} 


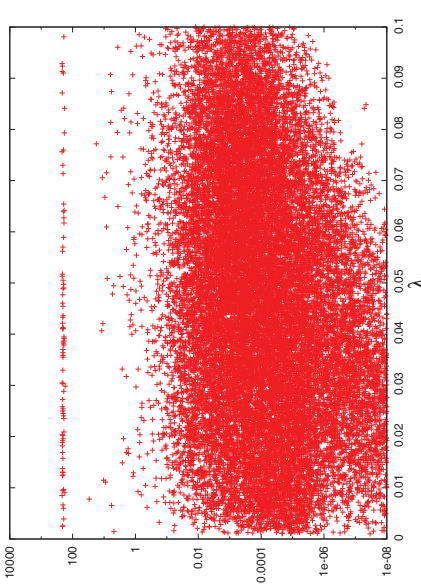

[q]] (² $\left.\left.{ }^{2} 1\right\}<-66\right) \circ$

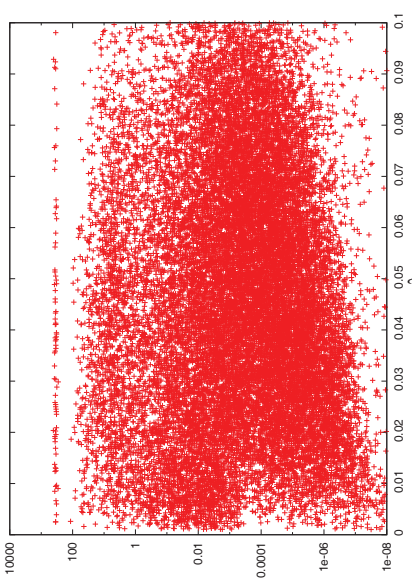

[q]] ('qqq<-66)॰

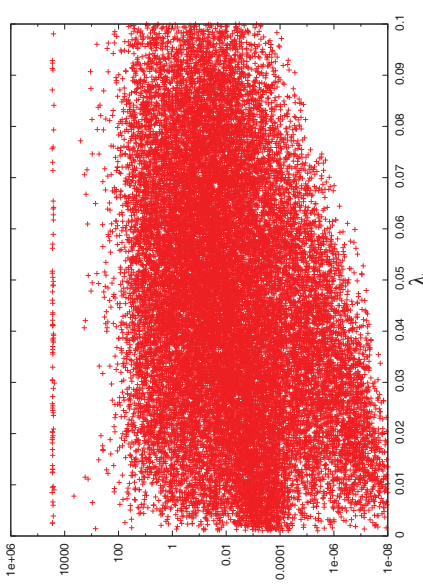

[qı] $\left({ }^{2}<<-66\right) \circ$

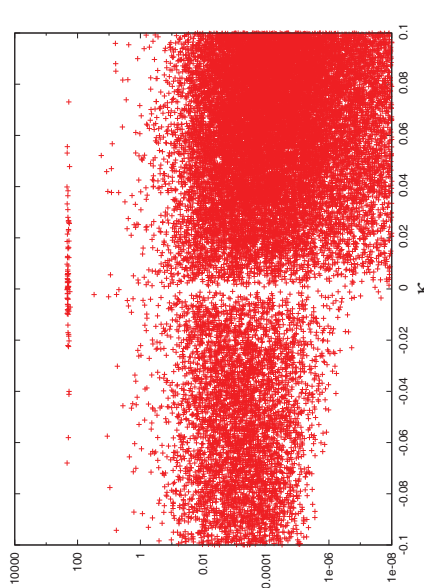

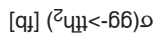

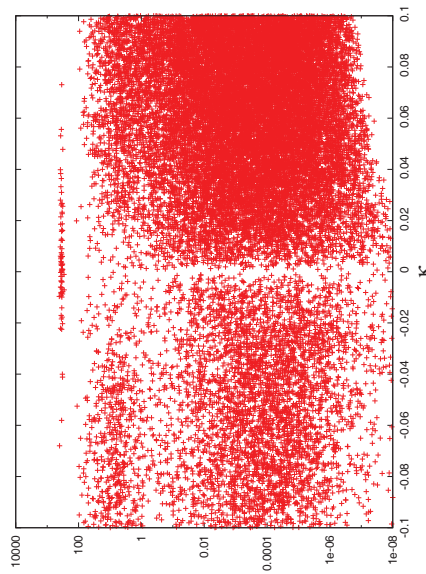

[q]] ('qqq<-66)॰

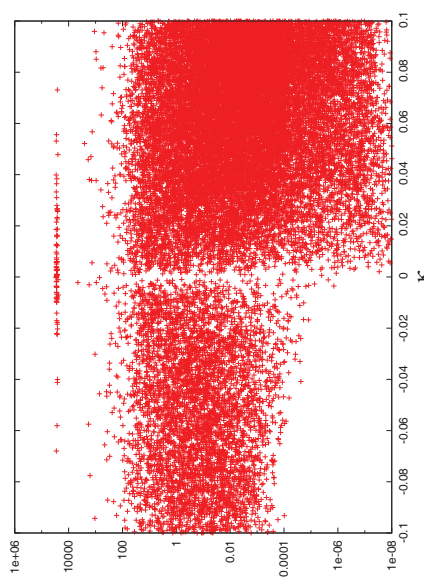

[q!] $\left({ }^{2} \mathrm{U}<-66\right)$ อ

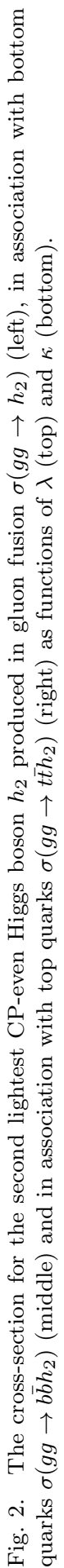




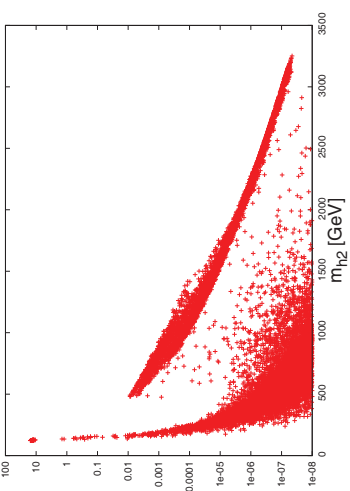

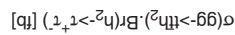

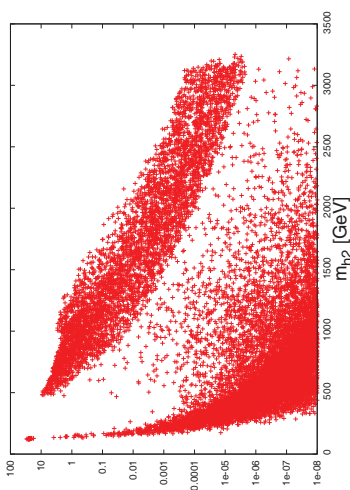

[q]] $\left(12+^{2<-}{ }^{2} u\right) \wedge g \cdot\left({ }^{2} u q q<-66\right) \rho$

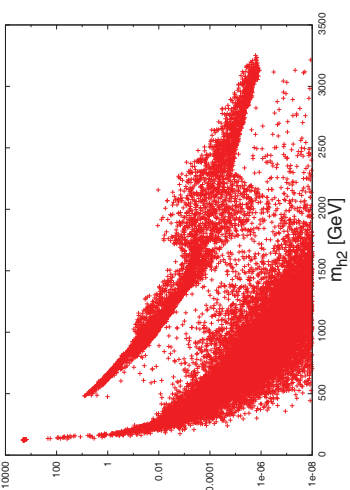

$[q]]\left(2_{+}+2<-z_{4}\right)+g \cdot\left(z_{y}<-66\right) \rho$

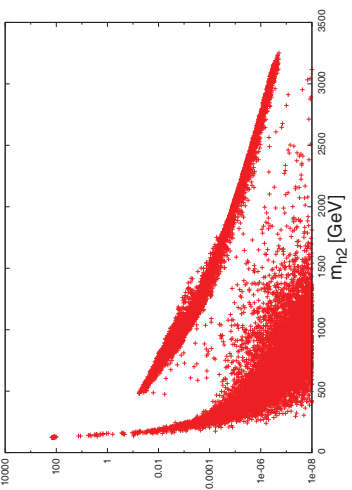

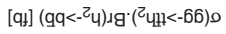

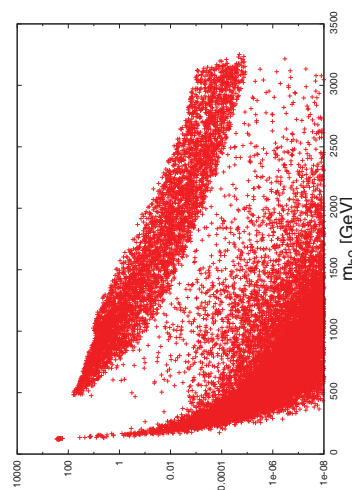

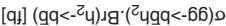

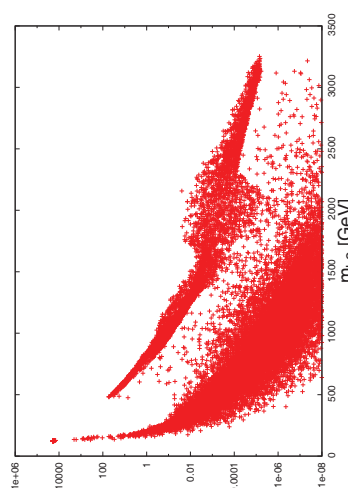

[q]] $\left(q q<-{ }^{2} y\right) \wedge g^{\prime} \cdot\left({ }^{2} u<-66\right) \rho$

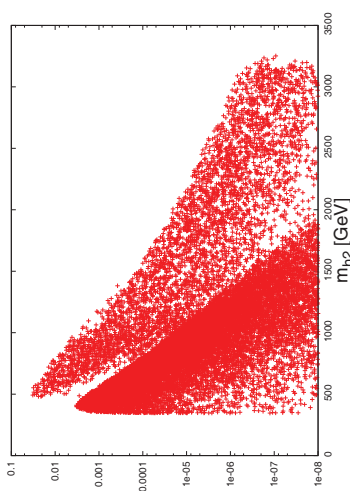

[q]] $\left(11<-{ }^{2} u\right) \wedge g \cdot\left({ }^{2} u[1<-66)>\right.$

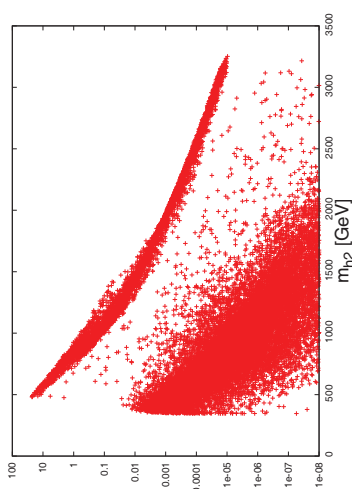

[q]] $\left.\left(11<-{ }^{2}\right)\right) \wedge g \cdot\left({ }^{2}\right.$ पqq $\left.<-66\right) \rho$

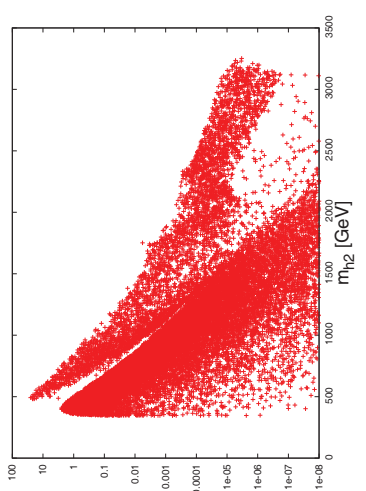

[q]] (11<- $\left.z_{4}\right) \wedge g \cdot\left({ }^{2} \mathrm{C}<-66\right)$ D

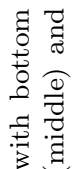

.

.

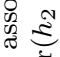

$\exists$ คี

苛 ลิ ?

$\uparrow+$

S)

a $\stackrel{1}{5}$

일

巳

을 छ

30 蛋

$\exists \stackrel{0}{0}$

రृ

苛

ㄴ.

응

웅

요요

50

iI

ㅇํㅇ

起

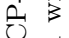

$+8$

D

000

च है है

ठ․

记 n

ㅇํㅇ

을 을

ปี छี శి

तิ

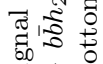

$\vec{\omega} \uparrow \triangleq$

E g

का के

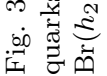


of the NMSSM parameter space in the mass range $m_{h_{2}} \gtrsim 480 \mathrm{GeV}$ where the production in association with bottom quarks can give the largest signal rate. This is because $h_{2}$ in this region has a large $H_{d}$ component, which can be substantial for large values of $\tan \beta$, so its couplings to down-type fermions are enhanced compared to the SM. In fact, $h_{2}$ production in association with bottom quarks has an extra advantage where the associated $b \bar{b}$ pair can be tagged, allowing a useful handle for background rejection. The production in association with a top-quark pair has only a considerable signal rate for the low mass range $m_{h_{2}} \lesssim 2 m_{W}$, giving maximum rates of about $15 \mathrm{fb}$ and $150 \mathrm{fb}$ in case of $\tau^{+} \tau^{-}$and $b \bar{b}$ final states, respectively, see the top left and middle left panels of the figure. In short, the size of the signal rates in some regions of the parameter space is quite large and that could help discovering $h_{2}$ in $\tau^{+} \tau^{-}$and $b \bar{b}$ final states. Also, $t \bar{t}$ final states may be exploited to discover $h_{2}$ in gluon fusion and in association with bottom-quark pair for $m_{h_{2}} \sim 480 \mathrm{GeV}$ or slightly above, though challenging due to large backgrounds and a complicated $t \bar{t}$ final state.

In Figs. $\underline{4}$ and $\underline{5}$, we present the production rates for $h_{2}$ into the SM bosonic particles. It is shown that $\gamma \gamma$ and $Z \gamma$ final states have only sizable signal rates for the low mass range $m_{h_{2}} \lesssim 2 m_{W}$, see Fig. 4 . It is clear that the largest production rate comes from gluon fusion channel, reaching maximum $70 \mathrm{fb}$ for the former and $45 \mathrm{fb}$ for the latter, see the left panels of the figure. The production rates for $h_{2}$ produced in association with bottom quarks and top quarks are quite small below $1 \mathrm{fb}$ level for both $\gamma \gamma$ and $Z \gamma$ final states. Although the signal rates for $\gamma \gamma$ and $Z \gamma$ final states are not so large, these final states offer clean signatures with manageable backgrounds at the LHC, so one may exploit these channels to discover $h_{2}$ in the low mass range especially in gluon fusion production channel. Figure $\underline{5}$ shows the signal rates in $W^{+} W^{-}$and $Z Z$ final states. It is shown that these rates reach up to $10,000 \mathrm{fb}$ for the former and $1000 \mathrm{fb}$ for the latter in gluon fusion channel, see the left panels of the figure. The $h_{2}$ production in association with both bottom and top quarks has approximately two orders of magnitude less than that of the gluon fusion production channel, see the middle and right panels.

It is interesting to note that $h_{2}$ decays into lighter Higgs pairs or into Higgs plus gauge boson are kinematically open in large areas of the NMSSM parameter space, see Fig. $\underline{6}$ which shows the signal rates in the presence of the following decays: $h_{2} \rightarrow a_{1} a_{1}$ (top), $h_{2} \rightarrow h_{1} h_{1}$ (middle) and $h_{2} \rightarrow Z a_{1}$ (bottom) for the gluon fusion production (left), the production in association with bottom quarks (middle) and the production in association with top quarks (right). It is shown from the figure that the largest signal rates also come from the gluon fusion production channel, reaching maximum about $1000 \mathrm{fb}$ for light $m_{h_{2}}$ in case of both $a_{1} a_{1}$ and $h_{1} h_{1}$ final

${ }^{9}$ The CMS collaboration published results of searches for a heavy neutral Higgs boson decaying into $W W$ and $Z Z$ bosons with up to $19.7 \mathrm{fb}^{-1}$ of data collected at a center-of-mass energy of $8 \mathrm{TeV}$. It was found that such a heavy Higgs boson with SM-like couplings and decays in the mass ranging from $145 \mathrm{GeV}$ to $1000 \mathrm{GeV}$ was excluded, see Ref. 51 . 
The second lightest CP-even Higgs boson signals in the NMSSM at the LHC

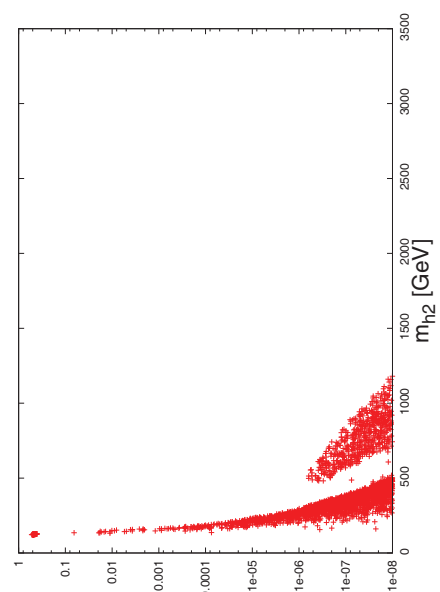

[q]] $\left(\mu<-{ }^{2} \mathrm{Y}\right) \wedge \cdot\left({ }^{2} \mathrm{Z}_{11<-66) \rho}\right.$

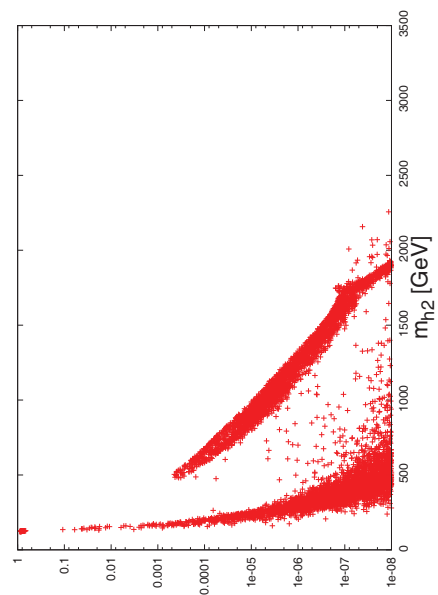

[q]] $\left(\mu<-\mathcal{L}_{\mathrm{u}}\right) \wedge \mathrm{g} \cdot\left({ }^{2} \mathrm{uqq}<-66\right) \rho$

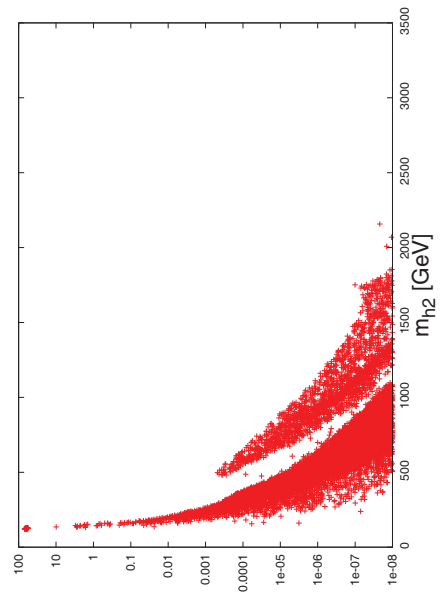

[q] $\left(\mu<-{ }^{2} \mathrm{U}\right) \wedge \mathrm{g} \cdot\left({ }^{2} \mathrm{U}<-66\right) \rho$

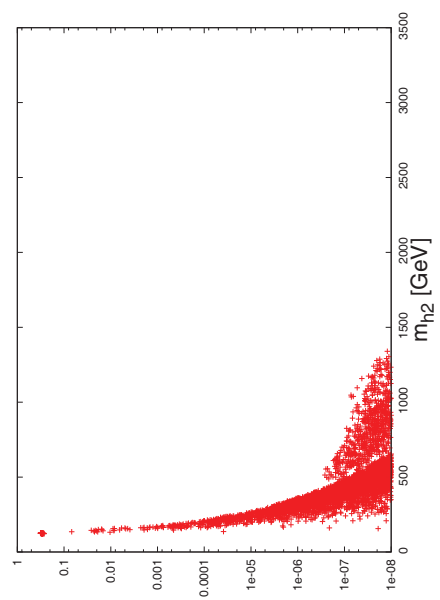

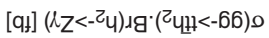

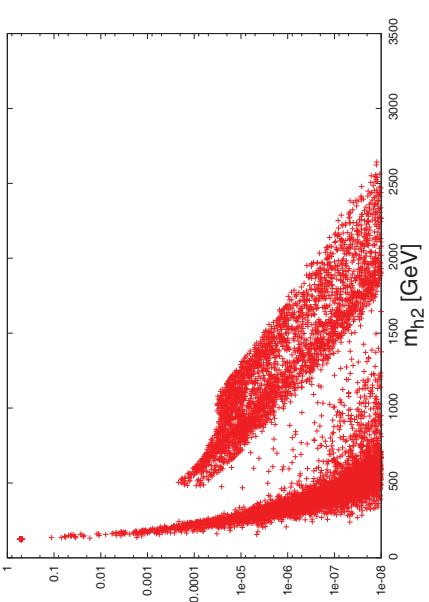

[q]] $\left(l_{Z}<-{ }^{2} u\right) \wedge q \cdot\left({ }^{2} u q q<-66\right) \rho$

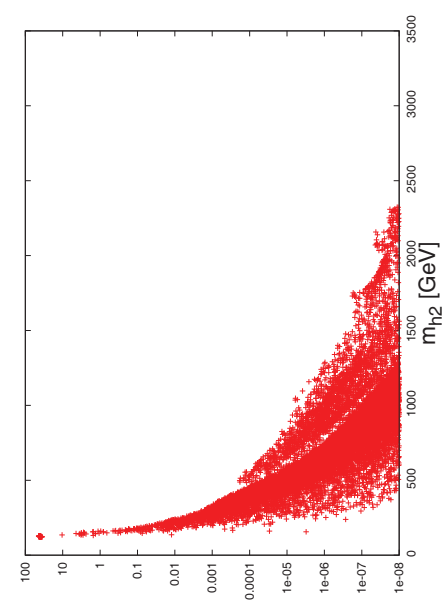

[qI] $\left(l_{\mathrm{Z}<-}{ }^{\mathrm{U}} \mathrm{u}\right) \wedge \mathrm{g} \cdot\left({ }^{\mathrm{Z}} \mathrm{U}<-66\right) \rho$

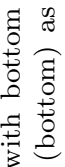

: $\overparen{\epsilon}$

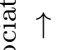

की ڤ

$\Xi \dot{n}$

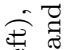

2ิ

సิ

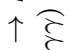

gु $\uparrow$

ㄷำ

을

记

₹.

음

평

$\nabla$

苛 胥

个 $\uparrow$

กิ

동

की

$\infty \frac{\infty}{60}$

讯

됴웅

(1)

क

(1)

o

$\pm$

$\because 0$

흐 ส

요.

记

๘

\$

ธิ 음

o

Ð

尔

ฮี่

क

ह

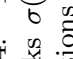

0. 


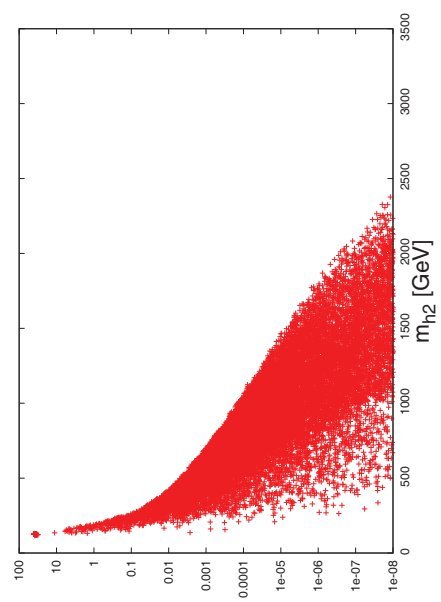

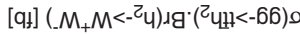

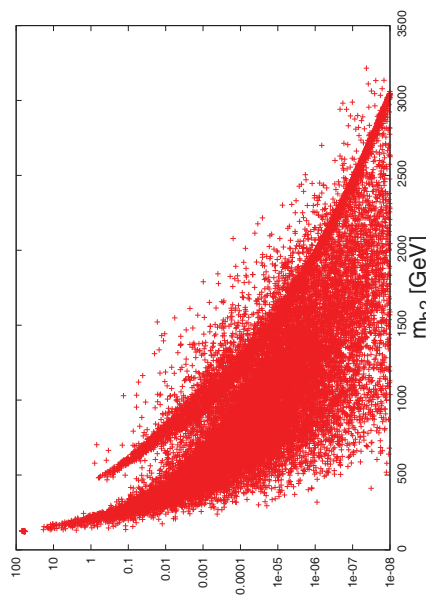

[q]] $\left(M_{+} M<^{2} \mathrm{C}\right) \wedge \mathrm{g} \cdot\left({ }^{2} \mathrm{uqq}<-66\right)$ ○

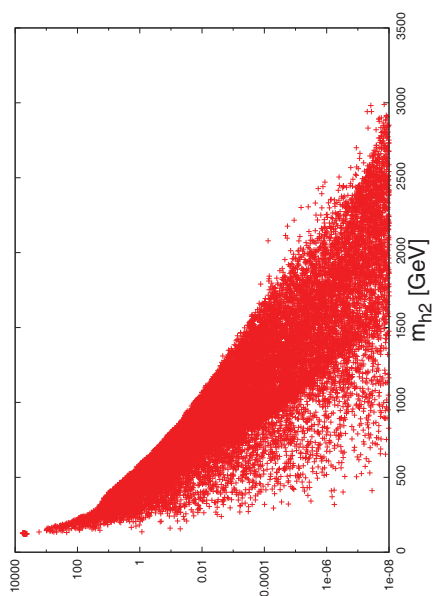

[q]] $\left(M_{+} M<--{ }^{2}\right) \wedge g^{\prime}\left({ }^{2} \mathrm{U}<-66\right)$ ○

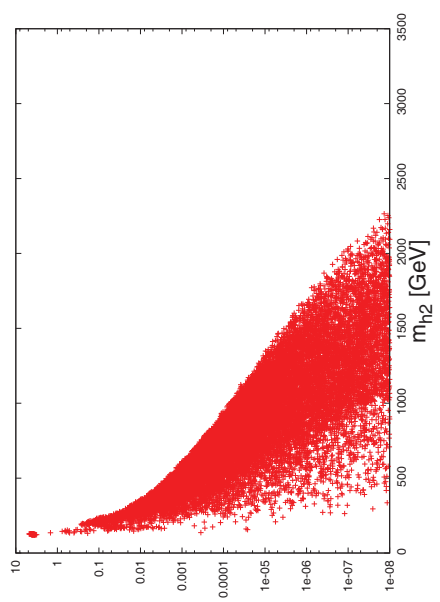

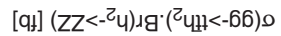

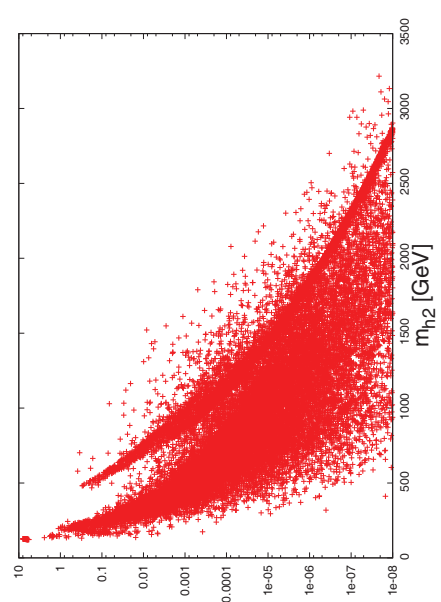

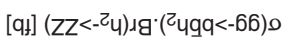

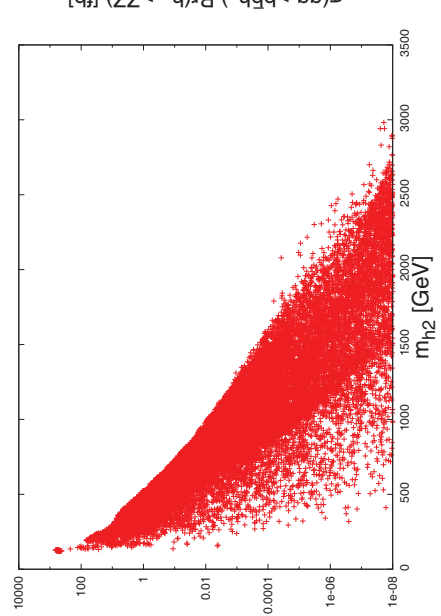

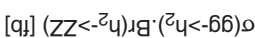

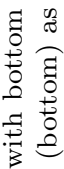

종

蛋个

की

$\Xi$ ตั

$\therefore \bar{\tau}$

$\stackrel{0}{=}$

ลิ

$\uparrow ?$

o:

:

ธี $\uparrow$

$\stackrel{\sqrt{0}}{\mathcal{E}}$

हี ติ

क्)

..

导

กิ

กิ

₹ $\uparrow$

o 5

$\begin{array}{ll}0 & 0 \\ 600 & 0 \\ .00 & 4\end{array}$

声

छี ซ

¿े 웅

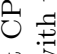

范

$\stackrel{ \pm}{ \pm}$ 。

.07

○.

光

$\circ$ I

D.

( )

के

우윰

ญ

雨

สี ิิ

5003

ज

छ

نे

$\dot{0}$ 


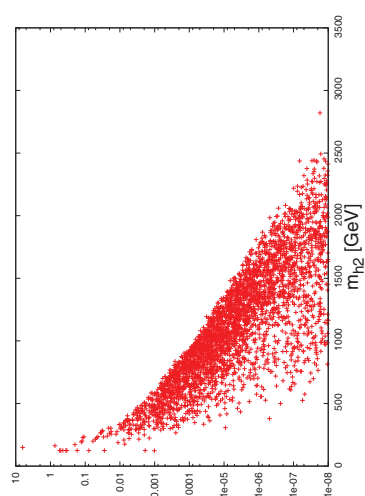

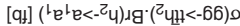

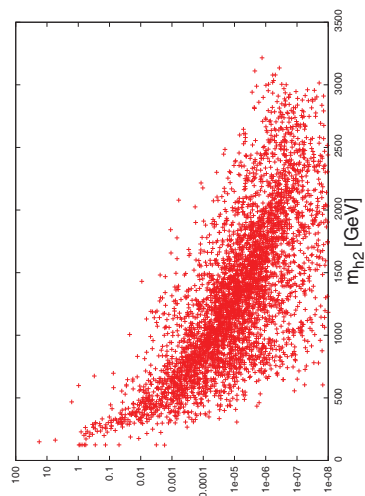

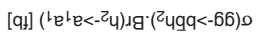

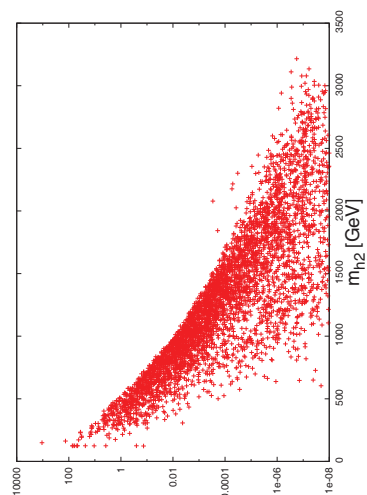

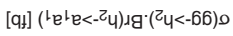

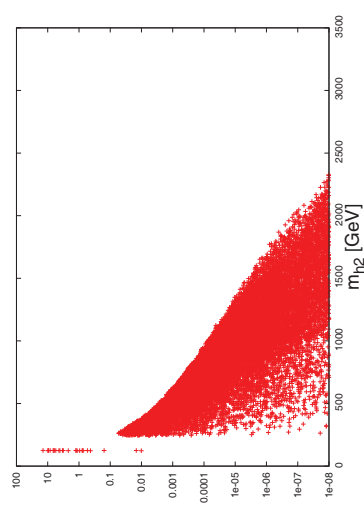

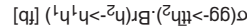

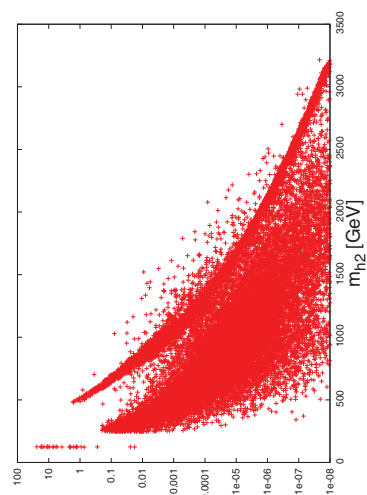

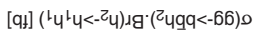

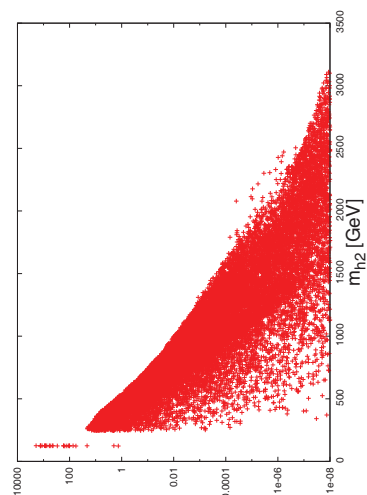

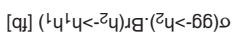

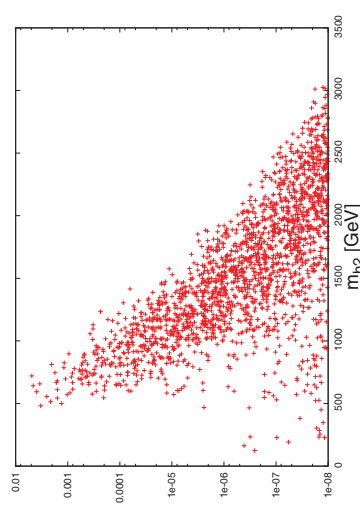

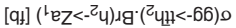

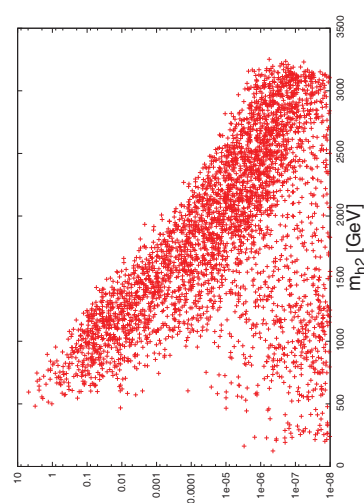

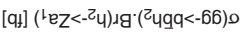

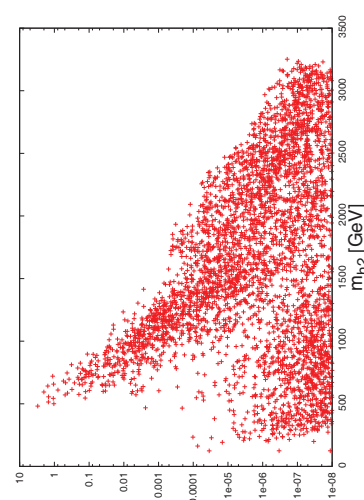

[q]] ( $\left.{ }^{\circ} \mathrm{e} Z<-\mathrm{C}_{\mathrm{C}}\right) \wedge \mathrm{g} \cdot\left(\mathrm{z}_{\mathrm{Y}}<-66\right) \rho$
픙

용

空劳

.

O

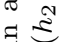

苗

$\stackrel{\rightleftarrows}{\stackrel{0}{\rightleftarrows}}$

(ิ)

$\uparrow$ ह

o 5

$\underbrace{5}_{b} \uparrow$

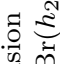

$\overbrace{}^{2}$

됴 है

f 코

$\exists .00$

$\mathrm{d} \Xi$

छิ

离 ¿

요

$\begin{array}{ll}0 & 0 \\ 0 & 0 \\ 0 & 0\end{array}$

$\infty$
$\infty$
0
0

ㄱํㅇ

Dิ

章

品.

8

00

च है ह

च

ర్ల

๑ สี

의

近司

छ छ

त्ञ

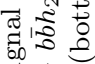

म

E

. $\begin{array}{r}0 \\ 0\end{array}$

它荠 
states and $5 \mathrm{fb}$ for $m_{h_{2}} \sim 480 \mathrm{GeV}$ in case of $Z a_{1}$ final state. The signal rates for $h_{2}$ production in association with both bottom and top quarks are quite small in most NMSSM parameter space except for small region where both $a_{1} a_{1}$ and $h_{1} h_{1}$ final states reach maximum signal rates of about $20 \mathrm{fb}$ for the production channel $g g \rightarrow b \bar{b} h_{2}$ (two top middle panels) and $10 \mathrm{fb}$ for the production channel $g g \rightarrow t \bar{t} h_{2}$ (two top left panels). The $Z a_{1}$ final state can only give sizable signal rates in the mass range $m_{h_{2}} \gtrsim 480 \mathrm{GeV}$, reaching up to $3 \mathrm{fb}$ for the production channel $g g \rightarrow h_{2}$ (bottom left panel) and $5 \mathrm{fb}$ for the production channel $g g \rightarrow b \bar{b} h_{2}$ (bottom middle panel). It is remarkable to note that $a_{1} a_{1}, h_{1} h_{1}$ and $Z a_{1}$ final states will further decay into quarks or leptons. The promising channels which may be exploited to discover $h_{2}$ are $2 \tau 2 b$ and $4 \tau$ final state as $4 b$ final state may be difficult to exploit because of the large background. Such extracted signals may require very large integrated luminosities of $300 \mathrm{fb}^{-1}$ or higher.

\section{Conclusions}

The discovered SM-like Higgs boson can be accommodated in the framework of the NMSSM. In this model, by assuming CP-conservation, there are seven Higgses: three CP-even Higgses $h_{1,2,3}\left(m_{h_{1}}<m_{h_{2}}<m_{h_{3}}\right)$, two CP-odd Higgses $a_{1,2}\left(m_{a_{1}}<\right.$ $m_{a_{2}}$ ) and a pair of charged Higgses $h^{ \pm}$. We have computed the cross-section for $h_{2}$ produced in gluon fusion, in association with a $b \bar{b}$ pair and in association with a $t \bar{t}$ pair in the following final states: $\tau^{+} \tau^{-}, b \bar{b}, t \bar{t}, \gamma \gamma, Z \gamma, W^{+} W^{-}, Z Z, a_{1} a_{1}$, $h_{1} h_{1}$ and $Z a_{1}$ at the LHC with $\sqrt{s}=14 \mathrm{TeV}$. It has been found that the size of the signal rates in some regions of the parameter space is quite large and that could help discovering the $h_{2}$ through $\tau^{+} \tau^{-}, b \bar{b}, \gamma \gamma$ and $Z \gamma$ final states in the low mass range $m_{h_{2}} \lesssim 2 m_{W}$. Also, it has been found that $W^{+} W^{-}$and $Z Z$ final states can give considerable signal rates for the mass range $m_{h_{2}} \lesssim 500 \mathrm{GeV}$. For most of the NMSSM parameter space, the dominant contribution to the $h_{2}$ production comes from the gluon fusion production channel. Furthermore, it has been noted that there exist some regions of the NMSSM parameter space where the production rates of the $h_{2}$ in the mass range $m_{h_{2}} \gtrsim 480 \mathrm{GeV}$ are quite sizable, and may be exploited to discover $h_{2}$ in $\tau^{+} \tau^{-}, b \bar{b}$ and $t \bar{t}$ final states through its production in association with $b \bar{b}$ pair, which has the dominant contribution to the $h_{2}$ production in this mass range, or through the gluon fusion channel.

Finally, we have calculated the signal rates of $h_{2}$, decaying into $a_{1} a_{1}, h_{1} h_{1}$ and $Z a_{1}$ final states. Although $h_{2}$ production rates are quite small in most of the NMSSM parameter space, it has been found that there exist some regions where the signal rates of $h_{2}$ are sizable and that could help discovering $h_{2}$ at the LHC in $a_{1} a_{1}, h_{1} h_{1}$ and $Z a_{1}$ final states, but such discovery may require very large integrated luminosities of $300 \mathrm{fb}^{-1}$ or higher.

\footnotetext{
${ }^{\mathrm{h}}$ The $h_{2}$ decays to SUSY particles, if kinematically possible, can be dominant, making searching for $h_{2}$ at the LHC very complicated.
} 


\section{Acknowledgment}

This work is funded by Taibah University, KSA.

\section{References}

1. H. P. Nilles, M. Srednicki and D. Wyler, Phys. Lett. B 120, 346 (1983).

2. J. M. Frere, D. R. T. Jones and S. Raby, Nucl. Phys. B 222, 11 (1983).

3. J. P. Derendinger and C. A. Savoy, Nucl. Phys. B 237, 307 (1984).

4. J. R. Ellis, J. F. Gunion, H. E. Haber, L. Roszkowski and F. Zwirner, Phys. Rev. D 39, 844 (1989).

5. M. Drees, Int. J. Mod. Phys. A 4, 3635 (1989).

6. U. Ellwanger, M. R. de Traubenberg and C. A. Savoy, Phys. Lett. B 315, 331 (1993).

7. S. F. King and P. L. White, Phys. Rev. D 52, 4183 (1995).

8. F. Franke and H. Fraas, Int. J. Mod. Phys. A 12, 479 (1997).

9. U. Ellwanger, M. R. de Traubenberg and C. A. Savoy, Nucl. Phys. B 492, 21 (1997).

10. M. Maniatis, Int. J. Mod. Phys. A 25, 3505 (2010).

11. U. Ellwanger, C. Hugonie and A. M. Teixeira, Phys. Rep. 496, 1 (2010).

12. CMS Collab. (S. Chatrchyan et al.), Phys. Lett. B 716, 30 (2012).

13. ATLAS Collab. (G. Aad et al.), Phys. Lett. B 716, 1 (2012).

14. CMS Collab. (S. Chatrchyan et al.), J. High Energy Phys. 1306, 081 (2013).

15. ATLAS Collab. (G. Aad et al.), Phys. Lett. B 726, 88 (2013).

16. U. Ellwanger, J. High Energy Phys. 1203, 044 (2012).

17. J. F. Gunion, Y. Jiang and S. Kraml, Phys. Lett. B 710, 454 (2012).

18. S. F. King, M. Muhlleitner and R. Nevzorov, Nucl. Phys. B 860, 207 (2012).

19. J. J. Cao, Z. X. Heng, J. M. Yang, Y. M. Zhang and J. Y. Zhu, J. High Energy Phys. 1203, 086 (2012).

20. D. A. Vasquez, G. Belanger, C. Boehm, J. Da Silva, P. Richardson and C. Wymant, Phys. Rev. D 86, 035023 (2012).

21. U. Ellwanger and C. Hugonie, Adv. High Energy Phys. 2012, 625389 (2012).

22. R. Benbrik, M. Gomez Bock, S. Heinemeyer, O. Stal, G. Weiglein and L. Zeune, Eur. Phys. J. C 72, 2171 (2012).

23. J. F. Gunion, Y. Jiang and S. Kraml, Phys. Rev. D 86, 071702 (2012).

24. K. J. Bae, K. Choi, E. J. Chun, S. H. Im, C. B. Park and C. S. Shin, J. High Energy Phys. 1211, 118 (2012).

25. K. Agashe, Y. Cui and R. Franceschini, J. High Energy Phys. 1302, 031 (2013).

26. K. Choi, S. H. Im, K. S. Jeong and M. Yamaguchi, J. High Energy Phys. 1302, 090 (2013).

27. K. Kowalska, S. Munir, L. Roszkowski, E. M. Sessolo, S. Trojanowski and Y.-L. S. Tsai, Phys. Rev. D 87, 115010 (2013).

28. S. F. King, M. Muhlleitner, R. Nevzorov and K. Walz, Nucl. Phys. B 870, 323 (2013).

29. T. Gherghetta, B. von Harling, A. D. Medina and M. A. Schmidt, J. High Energy Phys. 1302, 032 (2013).

30. M. Badziak, M. Olechowski and S. Pokorski, J. High Energy Phys. 1306, 043 (2013).

31. S. Moretti and S. Munir, Adv. High Energy Phys. 2015, 509847 (2015).

32. J. Cao, X. Guo, Y. He, P. Wu and Y. Zhang, Phys. Rev. D 95, 116001 (2017).

33. B. Das, S. Moretti, S. Munir and P. Poulose, Eur. Phys. J. C 77, 544 (2017).

34. U. Ellwanger, Eur. Phys. J. C 71, 1782 (2011).

35. S. F. King, M. Muhlleitner, R. Nevzorov and K. Walz, Phys. Rev. D 90, 095014 (2014).

36. S. Baum, K. Freese, N. R. Shah and B. Shakya, Phys. Rev. D 95, 115036 (2017). 
37. C. Beskidt, W. de Boer and D. I. Kazakov, arXiv:1712.02531 [hep-ph].

38. S. P. Das, J. Fraga and C. Avila, arXiv:1712.04395 [hep-ph].

39. S. Baum, M. Carena, N. R. Shah and C. E. M. Wagner, arXiv:1712.09873 [hep-ph].

40. M. Almarashi, arXiv:1801.06777 [hep-ph].

41. CMS Collab. (S. Chatrchyan et al.), Phys. Lett. B 722, 207 (2013).

42. CMS Collab. (V. Khachatryan et al.), J. High Energy Phys. 1511, 071 (2015).

43. CMS Collab. (S. Chatrchyan et al.), Phys. Rev. Lett. 106, 231801 (2011).

44. CMS Collab. (S. Chatrchyan et al.), Phys. Lett. B 713, 68 (2012).

45. ATLAS Collab. (G. Aad et al.), J. High Energy Phys. 1302, 095 (2013).

46. CMS Collab. (V. Khachatryan et al.), J. High Energy Phys. 1410, 160 (2014).

47. ATLAS Collab. (G. Aad et al.), J. High Energy Phys. 1411, 056 (2014).

48. ATLAS Collab. (M. Aaboud et al.), Eur. Phys. J. C 76, 585 (2016).

49. ATLAS Collab. (M. Aaboud et al.), J. High Energy Phys. 1801, 055 (2018).

50. CMS Collab. (A. M. Sirunyan et al.), arXiv:1803.06553 [hep-ex].

51. CMS Collab. (V. Khachatryan et al.), J. High Energy Phys. 1510, 144 (2015).

52. ATLAS Collab. (G. Aad et al.), Eur. Phys. J. C 76, 45 (2016).

53. J. E. Kim and H. P. Nilles, Phys. Lett. B 138, 150 (1984).

54. R. D. Peccei and H. R. Quinn, Phys. Rev. Lett. 38, 1440 (1977).

55. R. D. Peccei and H. R. Quinn, Phys. Rev. D 16, 1791 (1977).

56. U. Ellwanger, J. F. Gunion and C. Hugonie, J. High Energy Phys. 0502, 066 (2005).

57. U. Ellwanger and C. Hugonie, Comput. Phys. Commun. 175, 290 (2006).

58. See http://www.th.u-psud.fr/NMHDECAY/nmssmtools.html.

59. D. J. Miller, R. Nevzorov and P. M. Zerwas, Nucl. Phys. B 681, 3 (2004).

60. A. Pukhov, arXiv:hep-ph/0412191. 